\title{
Cuidado de enfermagem na ótica das gestantes de alto risco
}

\section{Nursing care from the perspective of pregnant women at high risk \\ Cuidado de enfermería en la óptica de las embarazadas de alto riesgo}

\section{Recebido: 21/11/2018 \\ Aprovado: 15/03/2019 \\ Publicado: 13/05/2019}

\section{Samuel Vareira Ferreira 1 Marilu Correa Soares ${ }^{2}$ Susana Cecagno ${ }^{3}$ Camila Neumaier Alves ${ }^{4}$ Tatiane Machado Soares ${ }^{5}$ Luiza Rocha Braga ${ }^{6}$}

0 presente estudo tem como objetivo conhecer a percepção das gestantes de alto risco quanto à assistência de enfermagem de um hospital de ensino. Trata-se de um estudo qualitativo e descritivo realizado em novembro de 2016 por meio de entrevista semiestruturada, individual e gravada com nove mulheres internadas, cuja análise foi realiza conforme proposta operativa de Minayo. Os dados foram agrupados em duas categorias: assistência de enfermagem na visão de mulheres que vivenciam a gestação de alto risco; e, sentimentos vivenciados pelas gestantes de alto risco. Os resultados apontam para a importância dos profissionais de enfermagem na assistência às gestantes, por proporcionarem apoio e atenção a suas demandas. A assistência de enfermagem neste hospital mostrou-se satisfatória diante do conceito daquelas gestantes, sendo percebido como de qualidade e humanizado, proporcionando respeito e a valorização diante das necessidades emanadas na internação.

Descritores: Gravidez de alto risco; Cuidados de enfermagem; Enfermagem.

This study aims to know the perception of the high-risk pregnant women related to nursing care in a teaching hospital. This is a qualitative, descriptive study conducted in November 2016 through semistructured interview, individually applied and recorded with nine women hospitalized, whose analysis was carried out according to Minayo's operative proposal. Data were grouped into two categories: nursing care in the view of women experiencing high-risk pregnancies; and feelings experienced by high-risk pregnant women. The results point to the importance of nurses in care for pregnant women, by providing support and attention to their demands. Nursing care in this hospital was satisfactory according to the concept of those pregnant women, being perceived in enough quality and humanized, providing respect and appreciation on the needs arising from the hospitalization.

Descriptors: Pregnancy, High-risk; Nursing care; Nursing.

El presente estudio lleva como objetivo conocer la percepción de embarazadas de alto riesgo cuanto a la asistencia de enfermería de un hospital de enseñanza. Se trata de un estudio cualitativo y descriptivo realizado en noviembre de 2016 a través de encuesta semiesctructurada, individual y grabada con nueve mujeres internadas, cuyo análisis fue realizado de acuerdo con proposición operativa de Minayo. Los datos fueron agrupados en dos categorías: asistencia de enfermería en la visión de mujeres que viven la gestación de alto riesgo; y, sentimientos vividos por las gestantes de alto riesgo. Los resultados apuntan a la importancia de los profesionales de enfermería en la asistencia a las embarazadas, por proporcionar apoyo y atención a sus demandas. La asistencia de enfermería en este hospital se enseñó satisfactoria ante al concepto de aquellas gestantes, siendo percibido como calidad y humanizado, proveyendo respeto y la valoración frente a las necesidades en la internación.

Descriptores: Embarazo de alto riego; Atención de enfermeria; Enfermeria.

1. Enfermeiro Assistencial no Hospital Nossa Senhora Aparecida, Camaquã/RS, Brasil. ORCID: 0000-0001-9942-7167 E-mail: mukavf@gmail.com

2. Enfermeira Obstetra. Mestre em Assistência de Enfermagem. Doutora em Enfermagem em Saúde Pública. Professora Associada da Faculdade de Enfermagem da Universidade Federal de Pelotas (UFPEL), Pelotas/RS, Brasil. ORCID: 0000-0001-9171-1083 E-mail: enfermeiramarilu@gmail.com

3. Enfermeira Obstetra. Mestre em Gestão da Qualidade dos Serviços de Saúde. Doutoranda em Enfermagem pela UFPEL, Pelotas/RS, Brasil. ORCID: 0000-0002-3953-0688 E-mail: cecagno@gmail.com

4. Enfermeira. Especialista em Cuidado Pré-Natal. Mestre e Doutora em Enfermagem. Docente do Centro Universitário Ritter dos Reis (UniRitter), Porto Alegre/RS, Brasil. ORCID: 0000-0002-6455-3689 E-mail: camilaenfer@gmail.com

5. Enfermeira. Especialista em Projetos Assistenciais. Mestre em Enfermagem. UFPEL, Pelotas/RS, Brasil. ORCID: 0000-0003-1822-6692 E-mail: tatibi_tati@yahoo.com.br

6. Enfermeira. Mestre em Enfermagem, Pelotas/RS Brasil. ORCID: 0000-0001-9303-9155 E-mail: luizarochab@gmail.com 


\section{INTRODUÇÃO}

A gestação de alto risco é identificada quando a vida ou a saúde da mãe e/ou do feto tem maiores probabilidades de ser comprometida em relação à média da população ${ }^{1}$. Os sentimentos dessas mulheres são ambivalentes e distintos, e frequentemente mesclam entre a felicidade $\mathrm{e}$ o medo, a preocupação, a passividade e a insegurança, além dos desarranjos no ritmo da família que são fomentados pelas restrições impostas pelo estado de risco ${ }^{2}$.

Nesse cenário, a atenção pré-natal tem sido preocupação constante do Ministério da Saúde (MS), que tem investido na formação de profissionais em todos os níveis do sistema de saúde, por meio de investimentos tecnológicos, incluindo diretrizes, normas e protocolos que norteiam a assistência obstétrica de qualidade. 0 cuidado de prénatal é priorizado, tendo como características essenciais a qualidade e a humanização no atendimento à gestante 3 .

Para tal, os serviços de saúde devem cuidar e acolher a mulher com dignidade, tendo um olhar crítico e consciente de que ela é sujeito de direito e não objeto passivo da atenção prestada ${ }^{4}$. Apesar disso, as taxas de morbimortalidade materna e neonatal ainda continuam elevadas, refletindo os problemas de acesso e de qualidade da assistência, ressaltando as desigualdades sociais e regionais, situações que geram vulnerabilidades e comprometem o exercício dos direitos reprodutivos ${ }^{5}$.

Neste sentido, um estudo mostrou que, apesar de as gestantes apresentarem necessidades de saúde de ordens física, social, psicoemocional e educacional, os profissionais priorizam o controle de aspectos físicos e obstétricos da gestação de alto risco por meio de ações curativopreventivas $^{4}$. Nesta perspectiva, o MS tem apontado que a assistência pré-natal pressupõe avaliação dinâmica das situações de risco e prontidão para identificar problemas de forma a poder atuar, a depender do problema encontrado, de maneira a impedir um resultado desfavorável, pois a ausência de controle no pré-natal pode incrementar o risco para a gestante ou o recém-nascido ${ }^{1}$.

Para o MS, o acompanhamento da gestante e da puérpera na Atenção Básica (AB) deve ser multiprofissional, com ênfase na participação do profissional de enfermagem $^{3}$. 0 pré-natal classificado como baixo risco pode ser realizado pelo enfermeiro, de acordo com o Decreto $\mathrm{n}^{\mathrm{o}}$ 94.406/87, que regulamenta a Lei de Exercício Profissional da Enfermagem ${ }^{6}$. Assim, a consulta de enfermagem torna-se oportuna para discutir sobre o organismo materno, fetal e do recém-nascido; deve ser sistematizada e estruturada, de modo que favoreça a promoção, proteção e manutenção da vida e melhoria da qualidade da assistência prestada à pessoa, família e comunidade?.

A realização de atividades educativas com gestantes implica no remodelamento da percepção e do enfrentamento diante de intercorrências, por refletir e interferir na troca de saberes, na transparência dos saberes, na critica e na promoção da saúde. Essas estratégias fortalecem a confiança entre as mulheres e os profissionais e, cria um ambiente harmonioso e seguro para o estabelecimento do cuidado ${ }^{8}$.

A gestação apesar de ser um fenômeno fisiológico, pode ser um potencializador de agravos a saúde das mulheres. Algumas intercorrências e complicações gestacionais são esperados, em decorrência de alterações de funcionalidades metabólicas, circulatórias, neurológicas e renais ${ }^{9}$.

Este estudo justifica-se pela necessidade de compreender os sentimentos das gestantes de alto risco em processo de hospitalização, de modo a oportunizar a equipe de enfermagem um desenho de uma assistência mais efetiva e que contemple a integralidade da atenção a essa população.

Assim, por entender a necessidade de atenção e cuidados diferenciados e de qualidade, este estudo foi norteado pela seguinte questão de pesquisa? Qual a percepção das gestantes de alto risco em relação à assistência de enfermagem prestada em um hospital de ensino? Neste 
contexto, o objetivo deste estudo foi conhecer a percepção das gestantes de alto risco quanto à assistência de enfermagem de um hospital de ensino.

\section{MÉTODO}

Trata-se de um estudo de caráter descritivo, com abordagem qualitativa. 0 cenário foi uma unidade materno-infantil de um Hospital Universitário do sul do Rio Grande do Sul.

Participaram do estudo nove gestantes com disgnóstico de gestação de alto risco internadas em novembro de 2016. No período de coleta de dados havia 11 gestantes internadas, destas, duas recusaram-se a participar do estudo, restando nove que consentiram em participar e atenderam aos critérios do estudo: estarem internadas na maternidade com diagnóstico de gravidez de alto risco; ter idade igual ou superior a 18 anos; autorizarem o uso de gravador durante as entrevistas; disponibilidade em participar do estudo; concordar com a divulgação e publicação dos resultados em meios acadêmicos e científicos.

A coleta de dados foi realizada por meio de entrevista semiestruturada, norteada por questões fechadas e abertas referentes ao tema do estudo. A análise e a interpretação dos dados foram fundamentadas na proposta operativa, que se caracteriza por dois níveis de interpretação: o primeiro compreender as determinações fundamentais da pesquisa, que foi mapeada na fase exploratória da investigação; e, o segundo nível denominou-se fase interpretativa e apresentou as etapas de ordenação e a classificação dos dados. Após, os dados categorizados foram discutidos através da proposta operativa de Minayo ${ }^{10}$.

Esta pesquisa foi aprovada pelo Comitê de Ética em Pesquisa da Faculdade de Medicina - UFPel sob o número de protocolo 60555316.5.0000.5317 na data de 01 de outubro de 2016.

\section{RESULTADOS}

\section{Caracterização das participantes do estudo}

As participantes do estudo foram nove mulheres com faixa etária entre 18 e 45 anos que estavam vivenciando gestação de alto risco no período de coleta dos dados. Os fatores prevalentes de internação foram: trabalho de parto prematuro, má formação fetal, crescimento intrauterino restrito, diabetes gestacional, hipertensão arterial, pré-eclâmpsia, cardiopatia, infecção do trato urinário e hipertireoidismo.

\section{Assistência de enfermagem na visão de mulheres que vivenciam a gestação de alto risco}

Quando questionadas sobre a assistência de enfermagem que receberam, a maioria demonstrou satisfação no atendimento, verbalizando-a com expressões positivas:

Eu gostei deles todos. Eu fui bem atendida. (P1)

o tratamento dos enfermeiros é muito bom. Muito bom mesmo. São atenciosos, eu não tenho nenhuma queixa. (P2)

Conversam de tudo: como eu estou me sentindo, o que eu estou precisando, qualquer coisa e chama. Tudo em questão de amparar. Medicação sempre no horário, alimentação também. (P9)

Além do apontado, as mulheres entrevistadas destacaram as informações que receberam por parte dos profissionais de enfermagem, o que corroborou para o conforto e segurança na assistência que receberam durante a internação:

Eu acho que eles me explicaram tudo [informações de saúde]. Eles explicaram bem tudo. (P1)

Ah, eu gostei, porque são bem atenciosos. Perguntam e explicam bem. Se a gente fica em dúvida eles retornam para explicar de novo. (P3)

Como forma de cuidado, o enfermeiro é observado com desempenho satisfatório no contexto do ambiente hospitalar:

Nossa, muito boa. Estou me sentindo muito bem amparada aqui dentro. Estou me sentindo como se eu estivesse em casa. (P9)

Das nove entrevistadas, quatro não falaram da assistência de enfermagem de forma evidente:

Eles se preocupam bastante. Eles estão sempre perguntando, procurando saber também. (P7)

Pra ver eu não estou sentindo tanta falta, todos eles são muito atenciosos, todos eles bem prestativos. Não precisa nem chamar, eles estão sempre na volta. (P9)

Apresenta-se, ainda, a percepção das 
gestantes sobre o processo de internação vivenciado. Algumas referem que esse processo de adoecimento e internação está interferindo na sua rotina de vida e de suas famílias:

Interfere bastante, porque eu não sou daqui. Sou do interior de Canguçu, tenho dois filhos em casa, mais um que é adotivo, tenho marido. (P5)

Por um lado é ruim estar aqui dentro, porque a gente tá fora de casa. (P7)

Sim, sinto saudade da casa e dos outros dois filhos. (P9) Tudo que a minha filha Sara tem sou eu. E eu agora tenho que internar sempre assim. Sempre eu venho fico uns dias e vou pra casa. (P2)

Eu não gostei, 146T, porque eu ia ficar longe do meu gurizinho, que está com a minha mãe e a minha irmã. É a primeira vez que eu deixei ele assim, sozinho. Sempre cuidei dele. (P3)

\section{Sentimentos vivenciados pelas gestantes de alto risco}

Apresentam-se nesta categoria os sentimentos vivenciados pelas gestantes de alto risco durante a internação hospitalar. Este processo está diretamente relacionado à vivência das mulheres e de suas famílias com a atual condição de saúde, podendo haver variações na forma como convivem e lidam com a situação:

$O$ nervosismo e o medo [diante da internação hospitalar]. (P1)

Eu tive medo. Eu fiquei muito chateada. E eu não esperei o resultado aqui, que peguei e fui pra casa. (P2) Eu choro, eu só choro. Entendeste? (P8)

Meio preocupada, me senti preocupada ao mesmo tempo tranquila, porque eu tava sendo bem amparada. Só fiquei muito nervosa, na hora fiquei muito nervosa, na hora fiquei pensando que eles não queriam me falar a verdade, que eu poderia ter antes da hora. (P9)

Contudo, apesar da hospitalização, observou-se neste estudo que as gestantes de alto risco e seus acompanhantes expressaram suas esperanças e aguardavam ansiosamente por um nascimento feliz e um bebê saudável. Este sentimento era o que os fazia enfrentar o presente, repleto de dúvidas, medos e ansiedades, mas com esperança de um futuro promissor:

A gente, queira ou não queira, a preocupação sempre fica, 146T. Os filhos na escola, aquela coisa toda, então é preocupante. Mas a gente tenta pegar e se acalmar. (P4)

Eu vou ter elas antes do tempo. Uma semana a mais aqui dentro da minha barriga é uma semana a menos dentro do hospital. Agora estou mais tranquila. (P9)

A minha mãe me ajudou muito, assim o pai deles me ajudou muito, mas ela parece que é a minha base, é o que preciso. Dela que eu tiro muita força. (P7)

Tenho o um marido que me ajuda bastante. Tem a minha sogra me ajuda bastante. E minha mãe também que ajuda a cuidar dos meus filhos, por tudo isso dá para me manter tranquila. (P9)

Outros sentimentos também emergiram, e saudade de casa e dos afazeres refletiram em uma espera maior pela recuperação do estado de saúde apresentado: Saudade de casa. Sinto saudade das pessoas que eu via lá na rua, pouco tempo, mas faz falta. Sinto saudade das pessoas da minha casa. (P6)

Sim, sinto saudade da casa e dos outros dois filhos. Mas estou tranquila porque essa no caso vai ser a minha última gestação. (P9)

\section{DISCUSSÃO}

Os resultados deste estudo demonstram que o perfil das mulheres estudadas corrobora com outros estudos realizados com gestantes que vivenciaram a gestação de alto risco no Brasil ${ }^{12}$. Houve predominio de mulheres adultas, de cor branca e economicamente ativas, além disso destaca-se que os fatores de risco com maior ocorrência foram 0 diabetes mellitus gestacional (quatro mulheres) e hipertensão arterial sistêmica (três mulheres), reforçando a importância de medidas preventivas através da identificação dos fatores de risco precocemente e o manejo adequado a fim de minimizar os danos a saúde materna e infantil.

As participantes do estudo foram nove mulheres com faixa etária entre 18 e 45 anos que estavam vivenciando gestação de alto risco no período de coleta dos dados. Os fatores prevalentes de internação foram: trabalho de parto prematuro, má formação fetal, crescimento intrauterino restrito, diabetes gestacional, hipertensão arterial, pré-eclâmpsia, cardiopatia, infecção do trato urinário e hipertireoidismo.

Assistir as mulheres com maior risco gestacional é permitir compreender e apreender, a partir de experiências individuais e coletivas, a vivência de eventos patológicos ou não médicos aos quais se encontram expostas, e que podem gerar vulnerabilidades a serem consideradas na boa assistência pré-natal ${ }^{12}$.

Observa-se a predominância de um ambiente harmonioso, cuidadoso e de confiança, que possibilitou a expressão e 
verbalização de suas apreensões e dúvidas, além de permear um sentimento de segurança diante do atendimento recebido.

Acredita-se que as gestantes de alto risco necessitam de cuidado e atenção interdisciplinar e avaliação individualizada de acordo com as necessidades e características específicas de cada gestante, além do reconhecimento de aspectos que se relacionam com o seu sentido de vida.

De acordo com o exposto nos resultados, destaca-se que a assistência de enfermagem precisa estar direcionada a amparar e focar o cuidado de forma a contribuir para a segurança, a tranquilidade e o enfrentamento da gestante quanto à situação em que ela se encontra ${ }^{8}$.

Acredita-se que a enfermagem tem uma atribuição fundamental no empoderamento das mulheres no tocante ao conhecimento oportunizado pelas ações de educação em saúde. A consulta de pré-natal e a internação hospitalar são cenários em que o enfermeiro pode desenvolver e fortalecer ações de cuidado, pois as mulheres demandam orientações e explicações sobre sua saúde e de seu filho.

A assistência para a gestante considerada de risco é um desafio no dia a dia da atenção à saúde, pois os altos índices de mortalidade materna preocupam não só a sociedade, mas também as autoridades ${ }^{13}$. Salienta-se que o cuidado deve iniciar no prénatal, o que corrobora em uma ferramenta de detecção precoce de fatores de risco que podem transformar uma gravidez de risco habitual em alto risco, possibilitando a intervenção em tempo oportuno para aqueles que são modificáveis.

Os profissionais de enfermagem têm um importante papel na metamorfose na assistencia a saúde da gestante, sendo imprescindível que estes incorporem nas suas práticas de trabalho conhecimentos científicos que atendam as reais necessidades psicossociais, rompendo com o modelo com centralizado na doença ${ }^{14}$.

Por isso, reforça-se que o cuidado com as gestantes não deve ser realizado de forma mecanicista. É necessário desenvolvê-lo de maneira que possibilite também a escuta e o diálogo na perspectiva da humanização. Necessita-se tornar o papel da enfermagem marcante e impactante, para que as pessoas sob esse cuidado efetivamente reconheçam os enfermeiros dentro da equipe de saúde.

Em decorrência da hospitalização, podem ocorrer alterações no ritmo familiar e problemas para as gestantes, como o afastamento da mulher de seu domicílio, dos familiares, das atividades profissionais e domésticas; adaptações da gestante ao novo ambiente e hábitos culturais; solidão, ansiedade, tédio, depressão e medo; sobrecarga de funções para alguns familiares - normalmente o marido, que passa a assumir o cuidado com os filhos e com a casa $^{2}$.

Neste sentido, muitas vezes não são dadas alternativas ou possibilidades de escolha de cuidado às gestantes além da hospitalização e do repouso, uma vez que a restrição do movimento é considerada uma das soluções para a sobrevivência do filho, causando-lhes sentimento de culpa quando não conseguem seguir a conduta proposta ${ }^{12}$.

Acredita-se que o processo de gestar deve ser compartilhado com a rede familiar de apoio, sendo o companheiro o sustento principal desta contextura da gestação, parto e nascimento. Quando a gestante e sua família estão bem orientadas e sincronizadas no cuidado e portam conhecimentos sobre o transcorrer desse processo gestacional, são capazes de se autoajudarem e serem proativas no cuidado, contribuindo, assim, para um desfecho positivo de maneira mais efetiva.

A educação é essencial na condução da gestação de alto risco, de modo que a enfermagem deve explorar a dimensão do educar, na perspectiva de auxiliar a gestante e seu acompanhante no sentido de serem protagonistas do processo de gestar, propiciando uma vivência mais tranquila, menos traumática e mais satisfatória ${ }^{2}$.

Com o conhecimento e o entendimento de suas reais necessidades e expectativas de saúde e bem-estar, as gestantes têm ficado menos preocupadas, e conseguem expressar sentimentos mais positivos. 0 apoio de familiares é 
fundamental, corroborando para que a gestante vivencie o processo de adoecimento com mais tranquilidade e segurança.

Nesta lógica de pensamento, a essência do trabalho do enfermeiro é o cuidar, processo que envolve contato próximo com o usuário, denotando assistir o ser humano em suas necessidades, envolvendo atos, comportamentos e atitudes que dependem do contexto e das relações estabelecidas entre usuário e profissional. Para que o cuidado seja de fato efetivo, o enfermeiro necessita ser empático, ofertar cuidados à gestante de forma que contemplem também suas expectativas. A atitude do enfermeiro pode significar muito para quem necessita de cuidados ${ }^{15}$.

A escuta e o diálogo são habilidades próprias dos seres humanos, sendo comum a concepção da escuta como apenas o ouvir, levando a acreditar que a escuta é instintiva. É uma ferramenta essencial para que o usuário seja atendido na perspectiva do cuidado como ação integral; por meio dela, é possível a construção de vínculos, a produção de relações de acolhimento, o respeito à diversidade e à singularidade no encontro entre quem cuida e quem recebe o cuidado ${ }^{12}$.

0 sentimento de medo pela sobrevivência de seu filho representou uma das maiores preocupações para as gestantes deste estudo. Outros estudos identificaram sentimentos semelhantes, com a prevalência do medo, da ansiedade, da tristeza e, também, da felicidade, sendo comum a elas a expressão do temor pela vida de seus filhos, uma vez que têm consciência de que as intercorrências na gravidez podem gerar agravos ao bebê2,13.

A vivência da gestação de alto risco caracteriza-se por um processo extremamente complexo, dinâmico, subjetivo e diversificado, podendo ser individual e social. É uma experiência única, que se estende ao companheiro, à família e à sociedade. Não é apenas um evento biológico, uma vez que envolve transformações fisiológicas, psicológicas, sociais, econômicas, culturais, espirituais e alterações de papéis, implicando na aceitação ou não da gravidez; aspectos também inerentes ao processo de gestação normal ${ }^{2,16}$.

Essas experiências vivenciadas vão além do processo de gestar. Elas arcam com a responsabilidade dos cuidados em relação ao filho, o que remete ao fato de que, culturalmente, a responsabilidade da criação, dos cuidados e da educação dos filhos ainda recai em grande parcela sobre a mulher.

É delegado à mulher o papel secundário na hierarquia familiar, mesmo quando a ela cabem não só os cuidados com os familiares e com a estrutura física da residência, mas também a provisão financeira da família. As mulheres atuais, assim como suas mães e avós, ainda relutam em abandonar o controle e poder que sempre tiveram, mantendo e reforçando a ideia de que a mãe é insubstituível no cuidado de seus filhos porque só ela, que os gerou e "pariu", sabe como desempenhar bem esta tarefa ${ }^{16}$.

Os depoimentos evidenciam que as gestantes, ao mesmo tempo em que diziam estar seguras e bem atendidas, queixavam-se de saudades e preocupações com os familiares. Ansiavam por voltar ao lar, conviver com os filhos e o companheiro, voltar à rotina e aos costumes, assumir seu espaço, o controle de seu corpo, de seu tempo e de sua vida.

Pode-se perceber que a internação hospitalar decorrente de complicações de uma gestação de alto risco tem implicado diretamente no estado geral dessas mulheres. Observou-se a predominância de mulheres despreparadas para vivenciar o processo patológico no gestar, e o sentimento de responsabilidade diante da condição fetal, que foi significativo.

Desse modo, a enfermagem, por estar diretamente e integralmente assistindo essas mulheres, tem um papel fundamental na garantia do seu bem-estar, que está alicerçado no diálogo, na confiança e no conforto. 0 apoio emocional, o estímulo à verbalização e à expressão dos sentimentos e vivências e a promoção da educação em saúde possibilitará a elas a vivência do processo de adoecimento de forma mais tranquila e confiante. 


\section{CONCLUSÃO}

0 presente estudo possibilitou conhecer a percepção de gestantes de alto risco sobre a assistência de enfermagem recebida. Evidenciou-se que a assistência de enfermagem neste hospital de ensino está sendo satisfatória diante do conceito das participantes. Em sua maioria, as gestantes estabeleceram vínculos, confiança e segurança durante o tempo em que estiveram internadas. Ainda, observou-se o reconhecimento da importância dos profissionais da enfermagem na assistência a essas mulheres, uma vez que proporcionam apoio e atenção a suas demandas.

Com relação aos sentimentos expressos pelas gestantes, reforça-se que os profissionais de saúde invistam no diálogo, para obterem resultados mais satisfatórios em suas atividades. Tal investimento pode tornar a assistência de melhor qualidade e humanizada, proporcionando o respeito e a valorização do cuidado prestado.

A hospitalização modifica a rotina dessas mulheres e de seus familiares, e a enfermagem pode colaborar no intuito de proporcionar, além do cuidado, o apoio e o envolvimento dos acompanhantes no processo de cuidar. É por meio da transformação desse cuidado que emerge a proatividade das gestantes, o que colaborará em uma atenção mais horizontalizada, efetiva, ética e responsável.

Assim, almeja-se que este estudo desperte reflexões aos profissionais de enfermagem que assistem as gestantes de alto risco, mostrando a relevância da assistência que valoriza os sentimentos singulares, as demandas originadas, construindo vínculo com as mulheres, de forma que se sintam seguras para vivenciar este período crítico em sua vida.

Uma limitação deste estudo foi 0 número restrito de participantes, o que dificultou a ampliação da análise e a discussão dos dados. É imprescindível que novas pesquisas sejam feitas nesta temática, uma vez que as ações assistenciais podem ser qualificadas e o cuidado de enfermagem remodelado para atender às expectativas e às necessidades dessa população.

\section{REFERÊNCIAS}

1. Ministério da Saúde (Br). Gestação de alto risco: manual técnico. Brasília, DF: Ministério da Saúde; 2010. 304p.

2. Silva MRC, Vieira BDG, Alves H, Rodrigues DP, Vargas GS, Sa AMP. A percepção de gestantes de alto risco acerca do processo de hospitalização. Rev Enferm UERJ. 2013; 21(2):792-7.

3. Ministério da Saúde (Br). Atenção ao prénatal de baixo risco. Brasília, DF: Ministério da Saúde; 2012. 320p.

4. Oliveira VJ, Madeira AMF. Interagindo com a equipe multiprofissional: as interfaces da assistência na gestação de alto risco. Esc Anna Nery Rev Enferm. 2011; 15(1):103-9.

5. Xavier RB, Jannottil CB, Silva KS, Martin AC. Risco reprodutivo e renda familiar: análise do perfil de gestantes. Ciênc Saúde Colet. 2013; 18(4):1161-71.

6. Conselho Federal de Enfermagem (Br). Decreto n. 94.406/87. Regulamenta a Lei n. 7.498, de 25 de junho de 1986, que dispõe sobre o exercício da Enfermagem [Internet]. Brasília, DF: COFEN; 1987 [acesso em 15 dez 2016]. Disponível em: http://www.cofen.gov.br/decreto-n9440687_4173.html

7. Dantas CN, Santos VEP, Tourino FS. A consulta de enfermagem como tecnologia do cuidado à luz dos pensamentos de Bacon e Galimberti. Texto \& Contexto Enferm. [Internet]. 2016 [acesso em $15 \mathrm{dez} 2016$ ]; 25(1):e2800014. Disponível em: http://www.scielo.br/pdf/tce/v25n1/01040707-tce-25-01-2800014.pdf

8. Camillo BS, Nietsche EA, Salbego C, Cassenote LG, Dal Osto DS, Bock A. Ações de educação em saúde na atenção primária a gestantes e puérperas: revisão integrativa. Rev Enferm UFPE On Line. [Internet]. 2016 [citado em $15 \mathrm{dez} 2016$ ]; 10(6):4894-901. Disponível em: https://periodicos.ufpe.br/revistas/revistae nfermagem/article/view/11270/12905

9. Varela PLR, Oliveira RR, Melo EC, Mathias TAF. Pregnancy complications in Brazilian puerperal women treated in the public and private health systems. Rev Latinoam Enferm. [Internet]. 2017 [citado em $15 \mathrm{dez}$ 2016]; 25:e2949. Disponível em: 
http://www.scielo.br/pdf/rlae/v25/01041169-rlae-25-e2949.pdf

http://dx.doi.org/10.1590/1518-

8345.2156.2949

10. Minayo MCS. O desafio do conhecimento: pesquisa qualitativa em saúde. 13ed. São Paulo: Hucitec; 2013. 406p.

11. Conselho Nacional de Saúde (Brasil). Resolução no 466 de 12 de dezembro de 2012. Trata de pesquisas em seres humanos e atualiza a resolução 196 [Internet]. D.O.U., Brasília, DF, 13 dez 2012 [citado em 15 dez 2016]. Disponível em: http://conselho.saude.gov.br/resolucoes/20 12/reso466.pdf

12. Oliveira DC, Mandú ENT. Mulheres com gravidez de maior risco: vivências e percepções de necessidades e cuidado. Esc Anna Nery Rev Enferm. 2015; 19(1):93-101. 13. Wilhelm LA, Alves CN, Demori CC, Silva SC, Meincke SMK, Ressel LB. Sentimentos de mulheres que vivenciaram a gestação de alto risco: estudo descritivo. Online Braz J Nurs. [Internet]. 2015 [citado em 16 dez
2017];14(3).

Disponível

em:

http://www.objnursing.uff.br/index.php/nur sing/article/view/5206/html_882

14. Costa PCP, Garcia APRF, Toledo VP. Acolhimento e cuidado de enfermagem: um estudo fenomenológico. Texto \& Contexto Enferm. 2016; 25(1):2-7.

15. Maynart WHC, Abuquerque MCS, Brêda MZ, Jorge JS. A escuta qualificada e o acolhimento na atenção psicossocial. Acta Paul Enferm. 2014; 27(4):300-3.

16. Silva LCM. Trabalho e família na percepção de mulheres provedoras. Gênero na Amazônia. 2012; 1(1):61-83.

CONTRIBUIÇÕES
Samuel Vareira Ferreira, Marilu Correa
Soares e Camila Neumaier Alves
contribuíram na concepção, delineamento,
análise, interpretação dos dados e redação.
Susana Cecagno, Tatiane Machado
Soares, Luiza Rocha Braga participaram na
redação e revisão crítica

\footnotetext{
Como citar este artigo (Vancouver)

Ferreira SV, Soares MC, Alves CN, Cecagno S, Soares TM, Braga LR. Cuidado de enfermagem na ótica das gestantes de alto risco. REFACS [Internet]. 2019 [citado em inserir dia, mês e ano de acesso]; 7(2):143-150. Disponível em: inserir link de acesso. DOI: inserir link do DOI.

Como citar este artigo (ABNT)

FERREIRA, S. V.; SOARES, M. C.; ALVES, C. N.; CECAGNO, S.; SOARES, T. M.; BRAGA, L. R. Cuidado de enfermagem na ótica das gestantes de alto risco. REFACS, Uberaba, MG, v. 7, n. 2, p. 143-150, 2019. DOI: inserir link do DOI. Disponível em: inserir link de acesso. Acesso em: inserir dia, mês e ano de acesso.

Como citar este artigo (APA)

Ferreira, S.V., Soares, M.C., Alves, C.N., Cecagno, S., Soares, T.M. \& Braga, L.R (2019). Cuidado de enfermagem na ótica das gestantes de alto risco. REFACS, 7(2), 143-150. Recuperado em: inserir dia, mês e ano de acesso de inserir link de acesso. DOI: inserir link do DOI.
} 